\title{
Alteraciones cognitivas en pacientes con hidrocefalia crónica del adulto ("normotensiva"). Propuesta de un protocolo para su evaluación clínica
}

\author{
B. Benejam; M.A. Poca*; C. Junqué**; E. Solana y J. Sahuquillo* \\ Unidad de Investigación de Neurotraumatología-Neurocirugía, Institut de Recerca Vall d’Hebron. Servicio de Neurocirugía y Unidad de \\ Investigación de Neurotraumatología-Neurocirugía*. Hospital Universitario Vall d'Hebrón, Universidad Autónoma de Barcelona. Departamento \\ de Psiquiatría y Psicobiología Clínica. Universitat de Barcelona**. Barcelona.
}

\section{Resumen}

La hidrocefalia crónica del adulto (HCA), o hidrocefalia a presión normal, se caracteriza por una alteración de la marcha, una incontinencia de esfínteres y un deterioro cognitivo progresivo, asociado a una dilatación variable del sistema ventricular. El tratamiento habitual consiste en la colocación de un sistema de derivación de líquido cefalorraquídeo (LCR). Dadas las características de los pacientes y el riesgo implícito de la cirugía, es fundamental realizar un diagnóstico correcto del síndrome para detectar a aquellos pacientes que pueden obtener un mayor beneficio del tratamiento. La valoración neuropsicológica de estos pacientes puede contribuir, de forma importante, al diagnóstico de la HCA, definiendo el perfil de deterioro cognitivo característico en estos enfermos. La valoración neuropsicológica permite, asimismo, determinar la eficacia del tratamiento quirúrgico y ayuda a detectar la coexistencia de otras causas de demencia. El objetivo de este estudio es definir el patrón de afectación cognitiva en pacientes con HCA y presentar el protocolo de valoración clínico, funcional y neuropsicológico que utilizamos en nuestro centro.

PALABRAS CLAVE: Hidrocefalia crónica del adulto. Hidrocefalia normotensiva. Deterioro cognitivo. Tests neuropsicológicos. Demencia.

Cognitive impairment in Normal Pressure Hydrocephalus (NPH). A proposal for clinical evaluation protocol.

\section{Summary}

Normal pressure hydrocephalus (NPH) is characterized by gait disturbance, urinary incontinence and dementia, and is associated with variable ventricular enlargement. The most accepted treatment of NPH is the placement of a cerebrospinal fluid shunt. Owing

Recibido: 11-12-07. Aceptado: 12-02-08 to the characteristics of the patients and the invasive nature of the surgical treatment, it is fundamental to detect those patients who could obtain a greater benefit from the treatment. Neuropsychological assessment of these patients could significantly contribute to a better diagnosis of NPH, determining a cognitive deterioration profile for these patients, allowing the assessment of treatment efficacy and helping to detect other additional causes of dementia. The aim of this study is to describe the cognitive deterioration profile of NPH patients and to present the clinical, functional and neuropsychological assessment protocol used in our hospital.

KEY WORDS: Adult Chronic Hydrocephalus. Normal pressure hydrocephalus. Cognitive impairment. Neuropsychological assessment. Dementia.

Introducción

La Hidrocefalia Crónica del Adulto (HCA) fue originalmente descrita por Hakim en 1964 con motivo de su tesis doctoral publicada en la Universidad Javierana de Bogotá, Colombia ${ }^{31,32}$. Este síndrome, más conocido como hidrocefalia normotensiva, se caracteriza, en su forma clínica completa, por la coexistencia de alteraciones de la marcha, incontinencia de esfínteres y un deterioro cognitivo progresivo. El cuadro se asocia a una dilatación variable del sistema ventricular y a una presión de líquido cefalorraquídeo (LCR) frecuentemente normal2,32. Las formas atípicas e incompletas son muy frecuentes.

El síndrome puede ser idiopático o secundario a otras afecciones cerebrales, como la hemorragia subaracnoidea, los traumatismos craneoencefálicos, las meningitis, etc. En ambos casos, el mecanismo fisiopatológico más aceptado es una alteración de los mecanismos de reabsorción del LCR asociado a alteraciones de las propiedades viscoelás-

Abreviaturas. EA: enfermedad de Alzheimer. FAB: Frontal Assessment Battery. FPH: fisuras perihipocámpicas. HCA: hidrocefalia crónica del adulto. HDS: HIV Dementia Scale. LCR: líquido cefalorraquideo. MDRS: Mattis Dementia Rating Scale. MMSE: Mini Mental State Examination. MPT: Motor Performance Test. NPH: hidrocefalia de presión normal 
ticas del parénquima encefálico. El tratamiento habitual consiste en la colocación de un sistema de derivación de LCR ventrículo-peritoneal o ventrículo-atrial. Cuando el diagnóstico es correcto, el cuadro clínico mejora de forma parcial o total. Sin embargo, el porcentaje de pacientes que mejoran después del tratamiento quirúrgico es muy variable y oscila en las series publicadas entre el 27 y el $96 \%$ de los $\operatorname{casos}^{4-7,9,33,57,62,75}$. Esta variabilidad se debe a diversos factores, como la inclusión de pacientes con hidrocefalias de diversa etiología, la coexistencia de otras causas de demencia, como la Enfermedad de Alzheimer (EA) o la demencia vascular ${ }^{76}$, la implantación de distintos tipos de válvulas y sistemas derivativos o la utilización de unos criterios de selección de los pacientes poco estrictos y, a menudo, excesivamente heterogéneos.

Dadas las características de estos pacientes (edad avanzada, factores de riesgo, comorbilidad asociada...) y el riesgo implícito del tratamiento quirúrgico, es fundamental realizar un diagnóstico adecuado del síndrome e identificar a aquellos pacientes que pueden obtener un mayor beneficio del tratamiento. Estudios recientes han demostrado la existencia de una alta prevalencia de Enfermedad de Alzheimer (EA) en pacientes con sospecha de una HCA (casos mixtos) ${ }^{26,68}$. Estos pacientes con cuadros mixtos suelen presentar una peor respuesta a la cirugía, que en ocasiones se limita a una mejoría de la marcha y de las alteraciones de esfínteres. Por este motivo, la correcta detección de estos casos mixtos es de gran relevancia clínica ya que va a definir en gran parte el pronóstico de las alteraciones cognitivas, permitiendo al médico informar y discutir con la familia las expectativas terapéuticas ${ }^{68}$. La exploración neuropsicológica puede ayudarnos a distinguir a los pacientes con una HCA "pura" de los pacientes que presentan asociada una demencia de tipo cortical, como ocurre en la EA y en las formas mixtas de $\mathrm{HCA}^{19,37,76}$.

El objetivo fundamental de este estudio es definir el patrón de afectación cognitiva de los pacientes con una HCA. También pretendemos presentar el protocolo de valoración clínico, funcional y neuropsicológico que utilizamos en el servicio de neurocirugía del Hospital Universitario Vall d'Hebron de Barcelona para el estudio de este tipo de pacientes.

\section{Características del deterioro cognitivo en la HCA}

Con frecuencia se ha utilizado el término "demencia" para describir el deterioro cognitivo asociado a la HCA, pero la utilización de este término no es del todo apropiada. Muchos enfermos presentan cuadros leves de afectación cognitiva que no cumplen con los criterios aceptados de demencia establecidos en la versión IV del Manual Diagnóstico y Estadístico de los Trastornos Mentales (DSM $I V)^{3,21,76}$. El deterioro psicológico en estos enfermos se
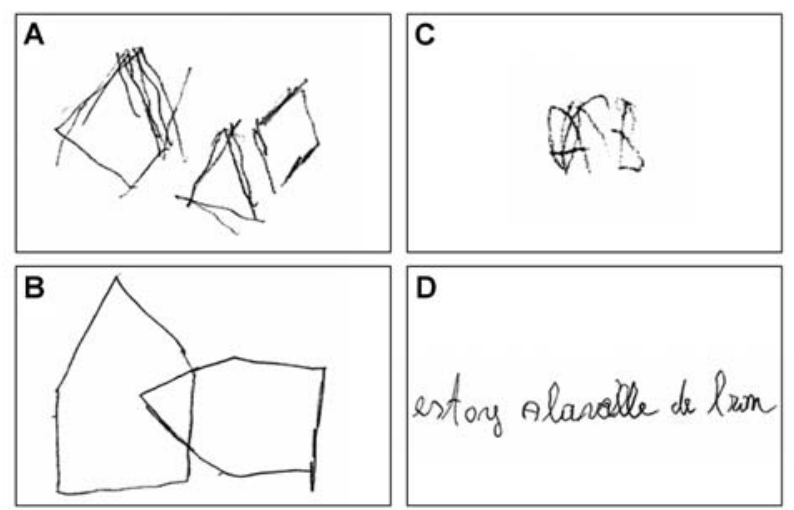

Figura 1. Copia de los pentágonos del Mini Mental (MMSE) y escritura de un paciente con hidrocefalia crónica del adulto. En $A$ y $C$, antes de la colocación de un sistema de derivación de líquido cefalorraquídeo. En $B$ y $D, 6$ meses después del tratamiento quirúrgico.

caracteriza por alteraciones tanto cognitivas como conductuales. El deterioro neuropsicológico suele debutar con una afectación de la memoria, con dificultades para recordar hechos recientes, y una alteración de la atenciónconcentración. A medida que va avanzando la enfermedad, se produce un empeoramiento progresivo de la memoria, que puede asociarse a episodios de fabulación (ver glosario en el anexo). Posteriormente se añaden otros déficits, como el enlentecimiento en la velocidad de procesamiento de la información, la alteración de las funciones ejecutivas, la incapacidad para manipular los conocimientos adquiridos, la desorientación temporal y espacial, la micrografía $\mathrm{y}$ el deterioro de las habilidades visuoconstructivas $\mathrm{y}$ visuoespaciales $^{18,76}$ (Figura 1). El déficit cognitivo de estos enfermos corresponde a un patrón de afectación de tipo fronto-subcortical, que ocurre en ausencia de signos de afasia, apraxia o agnosia, características típicas de la disfunción cerebral de tipo cortical ${ }^{12,18,30,72,76}$. Cuando éstos ocurren, suelen ser secundarios a un déficit de atención y suelen desaparecer parcialmente después de la colocación de una válvula. Si persisten o empeoran después de la intervención quirúrgica, deberá sospecharse la presencia de una demencia de tipo cortical concomitante.

A nivel conductual puede aparecer una disminución de la espontaneidad o de la iniciativa, una falta de interés por las actividades habituales y apatía e indiferencia hacia el medio, que en casos avanzados pueden llegar a sumir al enfermo en un estado de mínima conciencia o mutismo acinético $^{12,76}$. Con menor frecuencia, también pueden observarse conductas de tipo agresivo u hosti1 ${ }^{15,30,76}$.

Algunos autores han descrito síntomas psiquiátricos que suelen ser poco frecuentes y pueden dificultar el diagnóstico de HCA, como la depresión, la ansiedad, la psicosis paranoide o los trastornos obsesivo compulsivos ${ }^{1,8,48,55,56}$. Cuando esta sintomatología aparece en un paciente mayor, 
asociada a un deterioro cognitivo y a una alteración de la marcha, debería descartarse siempre el diagnóstico de $\mathrm{HCA}^{56}$. Larsson y cols. ${ }^{46}$ estudiaron a 72 pacientes con HCA de diversa etiología, y observaron que todos los pacientes mostraban alguno de los síntomas neuropsiquiátricos descritos por Lindqvist y Malmgren ${ }^{48,49}$, siendo los más frecuentes: 1) el "Trastorno Asteno-Emocional", en el que predomina una alteración de la atención y de la concentración, 2) el "Trastorno Emotivo-Motivacional", que se caracteriza por una disminución de la motivación, aplanamiento emocional e indiferencia hacia el medio y 3) el "Trastorno asociado a Somnolencia-Sopor-Coma", en el que se observa una disminución del nivel de vigilancia $^{46,48,73}$. Después de la intervención quirúrgica, el 78\% de los pacientes de este estudio mostraron una mejoría significativa de estos síntomas ${ }^{46}$.

\section{¿Qué cambios cognitivos podemos esperar después del tratamiento en los pacientes con una HCA?}

Los cambios postquirúrgicos más significativos después de la colocación de un sistema de derivación de LCR se han observado en la marcha y en el control de esfínteres ${ }^{21,29,57}$. El grado de mejoría en la función cognitiva suele ser menor y ocurre de forma más lenta ${ }^{40,61}$. Sin embargo, actualmente siguen existiendo controversias acerca de la magnitud de los cambios esperables en las funciones cognitivas después de la intervención quirúrgica. En diferentes series, se han descrito mejorías entre el $0-67 \%$ de los pacientes ${ }^{7,18,30,38,52,57,61,67,71,72}$. La memoria verbal diferida y las funciones ejecutivas son los dos dominios cognitivos que mejoran con mayor frecuencia ${ }^{18,19}$. En el estudio de Duinkerke y cols. ${ }^{18}$, el $80 \%$ de los pacientes mejoró en tareas de aprendizaje verbal, mientras que el 50\% lo hizo en pruebas de funciones ejecutivas. Otros estudios han mostrado mejorías significativas en la atención, concentración, memoria verbal y no verbal, velocidad motora, habilidades aritméticas, lenguaje y habilidades comunicativas, habilidades espaciales y constructivas ${ }^{46,70}$. Por el contrario, en un estudio prospectivo de 151 pacientes con HCA, Marmarou y cols. ${ }^{52}$ objetivaron cambios significativos en un único test neuropsicológico (memoria verbal a largo plazo) después de la realización de un drenaje lumbar externo. Una crítica importante a este trabajo es la utilización de instrumentos de evaluación neuropsicológica inapropiados para detectar los cambios neurocognitivos que se producen en estos enfermos ${ }^{19}$. También hay que considerar que la mejoría clínica después de un drenaje externo diagnóstico es, cuando ocurre, limitada.

Según algunos autores, la mejoría de los déficits cognitivos estaría relacionada con una mejoría en el sistema de activación (arousal) cerebral, a un decremento de la somnolencia diurna y a un aumento de la iniciativa ${ }^{13,73}$.
En el estudio de Tullberg y cols. ${ }^{73}$ la mejoría de estos síntomas se relacionaba con un aumento del flujo sanguíneo cerebral en el tálamo, en el hipocampo y en el córtex de asociación frontal. Este aumento del flujo es probablemente secundario a los cambios producidos en el sistema activador reticular ascendente.

Algunos autores han sugerido que la mejoría postquirúrgica está influenciada por la extensión de la afectación cognitiva previa a la intervención. Thomas y cols. ${ }^{71}$ observaron que los pacientes con mejores puntuaciones en las pruebas de memoria verbal inmediata y diferida de la escala de memoria de Weschler ${ }^{78}$ y/o mejores resultados en el aprendizaje y evocación de una lista de palabras, presentaban una mayor probabilidad de mejorar después de la colocación de una válvula.

Según Iddon y cols. el deterioro cognitivo de los pacientes con HCA no es del todo reversible ${ }^{38}$. A medida que aumenta el tiempo de evolución del proceso, el daño cerebral puede extenderse hasta afectar a la sustancia blanca subcortical y a la sustancia gris cortical, causando una alteración grave de la función mental. En un subgrupo de pacientes con HCA, estos autores observaron mejorías postquirúrgicas significativas en escalas globales de demencia, pero no observaron cambios en los tests neuropsicológicos sensibles a la afectación del lóbulo frontal, concluyendo que la alteración de estos circuitos persiste después del tratamiento quirúrgico ${ }^{38}$.

\section{Coexistencia con otras demencias. El problema de los casos mixtos}

En un paciente de edad avanzada con deterioro cognitivo y aumento de tamaño del sistema ventricular puede resultar difícil distinguir entre una HCA y una atrofia cerebral secundaria a la Enfermedad de Alzheimer (EA). Por otra parte, diversos autores han demostrado una alta prevalencia de EA en pacientes con sospecha de una $\mathrm{HCA}^{26,68}$. Golomb y cols. ${ }^{26}$ realizaron biopsias corticales en 56 pacientes con diagnóstico de HCA que fueron intervenidos quirúrgicamente mediante la colocación de un sistema de derivación de LCR. El 41\% de estos enfermos presentaba placas neuríticas características de la EA. En los pacientes que presentan un mayor grado de demencia (puntuación en la escala $\operatorname{GDS}^{63}$ mayor o igual a 6), la prevalencia de la EA puede incrementarse hasta un $75 \%{ }^{26}$. En el estudio de Savolainen y cols. ${ }^{68}$, los pacientes con una HCA y biopsia cerebral positiva para la EA presentaban peores resultados postquirúrgicos que los pacientes diagnosticados únicamente de HCA. Los pacientes que presentan estos cuadros mixtos pueden mejorar de forma significativa la marcha y el control de esfínteres, pero no suelen mejorar de forma significativa en las pruebas psicométricas ${ }^{26,71}$.

Otra causa frecuente de demencia en pacientes de 

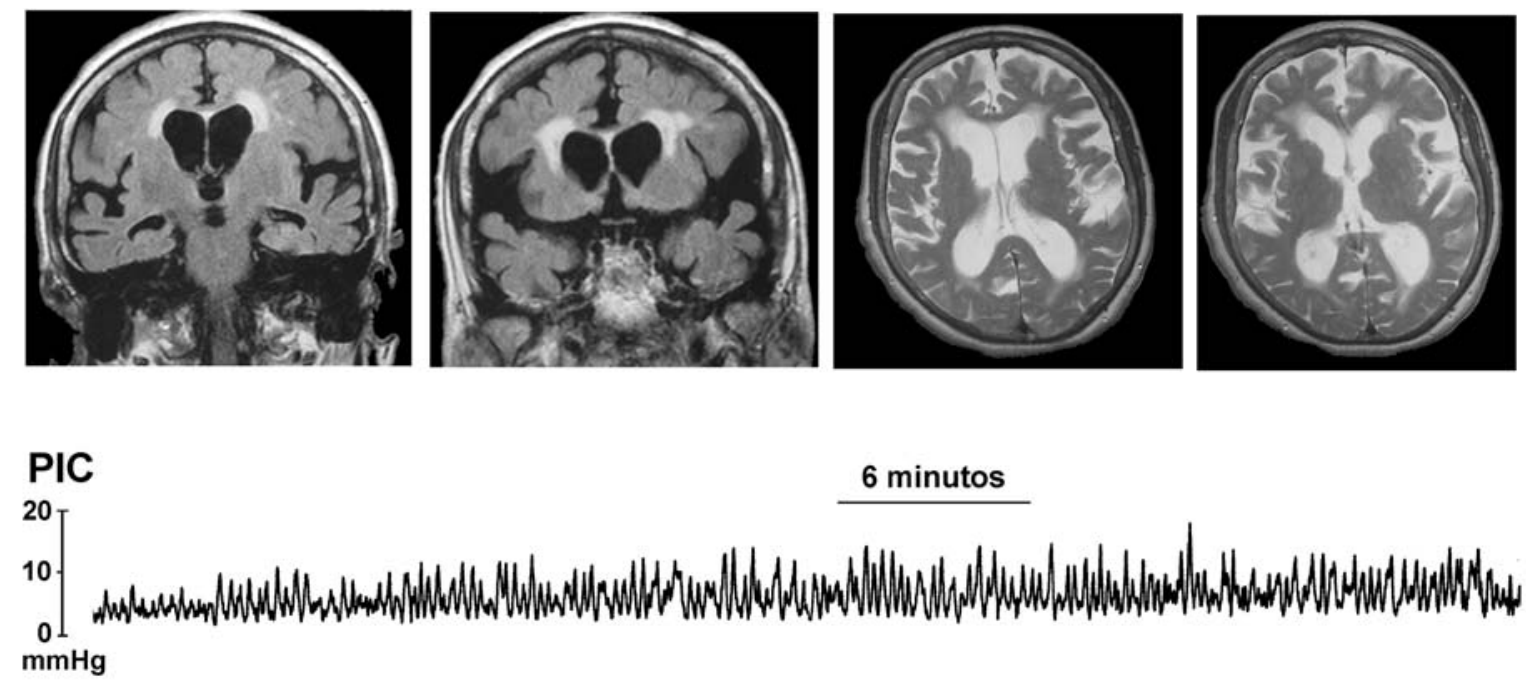

Figura 2. Imagen de RM de una paciente con una HC'A (Indice de Evans = 0,33), sin factores de riesgo de enfermedad cerebrovascular, en el que se observan hiperintensidades periventriculares y en sustancia blanca subcortical (flechas). Esta paciente presentaba alteraciones de la marcha que no habian respondido al tratamiento con L-Dopa, alteraciones cognitivas, cambio de carácter e incontinencia urinaria y fecal. En el registro gráfico de la presión intracraneal (PIC) se detectaron ondas $B$ de alta $(>10 \mathrm{mmHg}$ ) y baja amplitud $(<10 \mathrm{mmHg}$ ) en un $73 \%$ del trazado.

edad avanzada es la enfermedad cerebrovascular. En la enfermedad de Binswanger se observa una afectación de la sustancia blanca subcortical periventricular (leucoaraiosis), que se asocia a fenómenos de isquemia crónica y que produce un cuadro de demencia muy similar a la observada en la $\mathrm{HCA}^{14,59}$ (Figura 2). La dificultad para realizar un diagnóstico diferencial entre ambas patologías aumenta, si consideramos que muchos de estos pacientes pueden presentar una asociación de ambas entidades. Boon y cols. ${ }^{9}$ establecieron incluso una teoría patogénica según la cual la alteración de las propiedades viscoelásticas del parénquima cerebral, junto con la alteración de la dinámica del LCR podrían ser la causa de la dilatación ventricular observada en la HCA.

Por tanto, la coexistencia de varios procesos en un mismo paciente incrementa la posibilidad de encontrar casos en los que el deterioro cognitivo tenga un origen múltiple. Sin embargo, incluso en estos casos, el diagnóstico y tratamiento de una alteración en la dinámica del LCR puede aportar una mejoría clínica, fundamentalmente en la marcha y en el control de esfínteres, lo que puede resultar en una mayor independencia del paciente para las actividades de la vida diaria y, por consiguiente, una mejoría considerable de la calidad de vida del paciente y la de su entorno familiar. La falta de mejoría cognitiva en pacientes que han mejorado la marcha después de la colocación de una válvula, o en los casos en los que la demencia es el síntoma predominante, deberá sospecharse la existencia de un proceso neurodegenerativo concomitante como la EA Es,26,28. $^{25}$

La exploración neuropsicológica no permite discriminar la HCA de otras causas de demencia subcortical, pero puede ayudarnos a distinguir los déficit cognitivos causados por una demencia subcortical de las alteraciones cognitivas asociadas a una demencia de tipo cortical ${ }^{11}$. El patrón de afectación cognitiva que se observa en los pacientes con una HCA pura y en estadios no avanzados es diferente al que ocurre en las demencias corticales como la EA. Los pacientes con EA presentan una dificultad en la codificación de la información y, por consiguiente, una alteración en el reconocimiento de la información ${ }^{34}$. En cambio, los pacientes con una HCA muestran una dificultad en la evocación de la información, con un reconocimiento normal o relativamente preservado ${ }^{18,19,76}$. El perfil cognitivo en la HCA se asemeja al de las demencias subcorticales, incluyendo enlentecimiento en el procesamiento mental, alteración de las funciones ejecutivas y apatía. En la EA se pueden ver síntomas corticales como afasia, apraxia o agnosia, los cuales raras veces se observan en los pacientes con una $\mathrm{HCA}^{19,37,57,59,76}$. En los casos en los que la HCA se encuentra en un estadio muy avanzado, va a ser difícil diferenciar ambas entidades, dado que a medida que aumenta el tiempo de evolución, el daño cerebral puede extenderse hasta afectar a regiones cerebrales más corticales.

El análisis de determinados signos radiológicos, como la atrofia del hipocampo, puede también contribuir a orientar el diagnóstico. Los pacientes con una EA muestran, de forma casi invariable, una atrofia del hipocampo y, por consiguiente, una dilatación de las fisuras perihipocámpicas $(\mathrm{FPH})^{24,25,28,36,39,42}$. Holodny y cols. ${ }^{36}$ observaron diferencias significativas en el grado de dilatación de las FPH y en el tamaño de los ventrículos laterales en pacientes con HCA y pacientes con EA. Los pacientes con HCA presentan FPH 

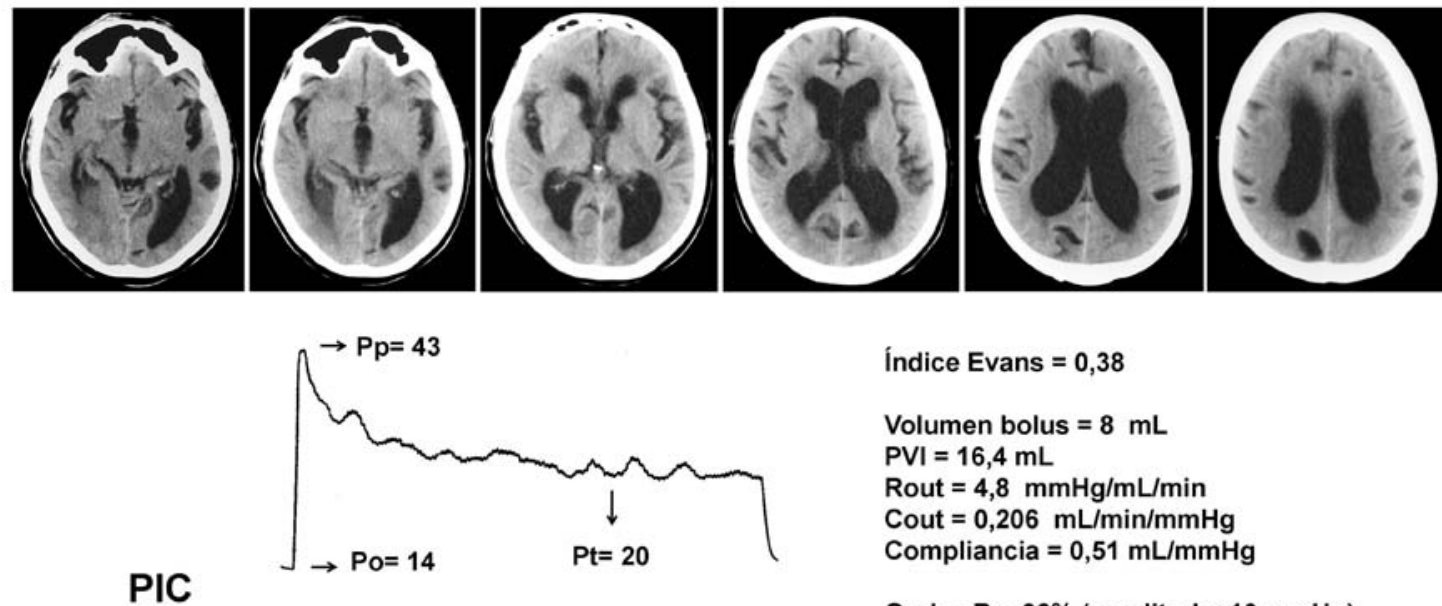

Índice Evans $=0,38$

Volumen bolus $=8 \mathrm{~mL}$

$\mathrm{PVI}=16,4 \mathrm{~mL}$

Rout $=4,8 \mathrm{mmHg} / \mathrm{mL} / \mathrm{min}$

Cout $=0,206 \mathrm{~mL} / \mathrm{min} / \mathrm{mmHg}$

Compliancia $=0,51 \mathrm{~mL} / \mathrm{mmHg}$

Ondas B $=32 \%$ (amplitud $<10 \mathrm{mmHg}$ )

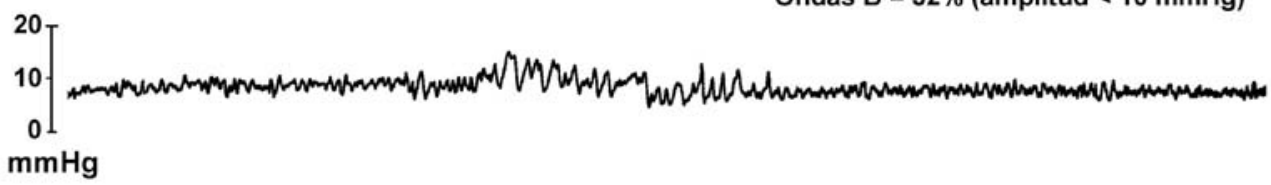

Figura 3. TC cerebral de un paciente con diagnóstico de HCA que objetiva la presencia de una dilatación del sistema ventrícular (Índice de Evans =0,38) en el contexto de unos surcos corticales aumentados y valles silvianos dilatados. En el centro se muestra el resultado de un estudio de la dinámica de LCR (test de bolus de Marmarou) que muestra un valor bajo del índice presión-volumen (PVI) y una resistencia a la reabsorción (Rout) del LCR normal ( $<5 \mathrm{mmHg} / \mathrm{mL} / \mathrm{min}$ ). La parte inferior de la imagen muestra un segmento del registro de la PIC del paciente, que constata la presencia de un trazado irregular con ondas de PIC patológicas (32\% de ondas B de baja amplitud). Seis meses después de la colocación de una derivación de LCR ventriculoperitoneal, el paciente presenta una mejoría de la marcha, del control de esfinteres, y de las funciones superiores.

pequeñas y unos ventrículos laterales muy dilatados, mientras que los pacientes con EA presentan unas FPH muy dilatadas y unos ventrículos menos dilatados. Estas diferencias pueden constatarse mediante la utilización de técnicas volumétricas automatizadas o mediante técnicas subjetivas de inspección visual $^{36}$. Por otro lado, la presencia de surcos corticales dilatados, indicadores de atrofia cortical, se ha utilizado como un criterio clásico que descarta el diagnóstico de HCA. Sin embargo, algunos estudios han demostrado que el agrandamiento de la cisura de Silvio y la dilatación de algunos surcos corticales es compatible con una buena respuesta a la derivación de $\mathrm{LCR}^{58,77}$. Se ha sugerido que estas dilataciones focales representan reservorios de LCR ubicados a nivel extraventricular $^{35,43}$. (Figura 3).

Finalmente cabe considerar que el análisis de los resultados conjuntos de la exploración neuropsicológica, de las técnicas de neuroimagen y del registro continuo de la presión intracraneal nos pueden ayudar a orientar el diagnóstico de HCA y a detectar la coexistencia de otros procesos como la demencia tipo Alzheimer o alteraciones vasculares.

Protocolo de estudio de los pacientes con sospecha de HCA

Según las guías de práctica clínica recientemente publicadas, no existe en la actualidad un estándar para la valoración de los resultados de la cirugía en la $\mathrm{HCA}^{44}$. Los protocolos de estudio postquirúrgicos suelen incluir escalas funcionales basadas en las actividades de la vida diaria del paciente, así como una evaluación psicométrica que permite la detección de cambios más sutiles en el dominio cognitivo del enfermo ${ }^{44}$.

La evaluación de un paciente que presenta un cuadro compatible con una HCA debería incluir la valoración de los tres componentes de la tríada clínica inicialmente descrita por Hakim y Adams ${ }^{2}$. Para ello se pueden utilizar escalas clínicas que valoren de forma cualitativa cada uno de estos síntomas. En el caso de la marcha, se han descrito en la literatura diversas pruebas que permiten cuantificar el grado de afectación del enfermo. Estas pruebas suelen incluir ítems que valoran la velocidad de la marcha, la longitud del paso, la habilidad para realizar giros, pruebas de equilibrio, etc. Para la valoración de las funciones cognitivas, se pueden utilizar pruebas de cribaje generales, que permitan definir la gravedad del deterioro cognitivo. Sin embargo, estas pruebas generales deberán complementarse con pruebas neuropsicológicas específicas para poder determinar cuáles son las funciones psicológicas más afectadas en cada caso. Además de la evaluación de funciones cognitivas específicas, es importante incluir alguna prueba 
que valore el cambio conductual de estos enfermos, y valorar los cuadros de apatía, la pérdida de espontaneidad, la irritabilidad, etc. Por último, deberían incluirse escalas para determinar cuál es la repercusión que esta enfermedad ejerce sobre las actividades de la vida diaria del paciente y cuál es el grado de dependencia de éste para la realización de las mismas.

\section{Tests de cribaje general. ¿Es útil el Minimental en estos} pacientes?

Para la valoración del deterioro cognitivo en pacientes con HCA se utilizan con frecuencia instrumentos generales como el Mini Mental State Examination (MMSE) ${ }^{22}$. Este test tiene la ventaja de ser breve, conocido por todos los clínicos, de fácil aplicabilidad y permite cuantificar el grado de demencia que presenta el paciente. El inconveniente de utilizar este test es que no proporciona datos cualitativos sobre cuáles son los dominios cognitivos alterados ${ }^{16}$. Además, el MMSE es inadecuado para detectar deterioros leves en la función cognitiva ${ }^{76}$, y ha demostrado no ser sensible al tipo de deterioro cognitivo que presentan los pacientes con $\mathrm{HCA}^{19,76}$. Como ya se ha comentado anteriormente, los pacientes con HCA muestran una afectación del sistema frontal ejecutivo, con importantes dificultades en la iniciación de las tareas y en su perseveración ${ }^{19}$. Un buen test de cribaje general debería valorar la integridad o disfunción de dicho sistema. Se ha demostrado que la puntuación del MMSE se correlaciona de forma significativa con el grado de atrofia del hipocampo, característico de la EA, y no con el grado de dilatación del sistema ventricular presente en los pacientes con una $\mathrm{HCA}^{25}$. El MMSE no es eficaz para diferenciar a los pacientes con deterioro cognitivo causado por la HCA de los pacientes que presentan cambios neuropatológicos sugestivos de $\mathrm{EA}^{54,67,68}$. Por tanto, cuando se decide utilizar este instrumento, útil para la valoración global de la función cognitiva, deberá hacerse en combinación con otras pruebas que sean sensibles a la patología que se está estudiando.

Entre las pruebas de cribaje general que han demostrado ser sensibles a la demencia causada por la afectación de los circuitos fronto-subcorticales, destacan los siguientes: 1) Mattis Dementia Rating Scale (MDRS) ${ }^{19,53}$, 2) Frontal Assessment Battery (FAB) ${ }^{17}$ y 3) HIV Dementia Scale $(\mathrm{HDS})^{60}$.

La MDRS es un test que puede administrarse en 15 minutos, es sensible al grado de demencia en los pacientes con HCA e incluye valores comparativos para una muestra de pacientes con $\mathrm{EA}^{19}$. El FAB es un instrumento que incluye 6 subtests que valoran la función del lóbulo frontal. Miyoshi y cols. han demostrado su sensibilidad para detectar el deterioro cognitivo de tipo subcortical al comparar los resultados obtenidos entre un grupo de pacientes con
HCA con otro grupo de pacientes con EA. Los pacientes con HCA muestran puntuaciones significativamente más bajas que los pacientes con EA en esta prueba ${ }^{54}$. La HDS es una prueba que ha demostrado discriminar entre pacientes que presentan un deterioro cognitivo de tipo subcortical y controles sanos de edad avanzada. Es, además, útil para detectar la presencia de un deterioro cognitivo incluso en pacientes sin demencia, es decir, con una puntuación en el MMSE $>23^{74}$.

\section{¿Cuándo valorar los resultados de la cirugía?}

No existe en la actualidad un consenso sobre cuál es el momento idóneo para valorar los resultados de la cirugía ${ }^{44}$. La comorbilidad no relacionada con la cirugía, como los accidentes cerebrovasculares isquémicos o hemorrágicos, o la aparición de otras causas de demencia, pueden influir en los resultados clínicos del paciente ${ }^{62,67}$. Además, la edad avanzada de estos pacientes hace que también puedan solaparse las repercusiones de otros procesos sistémicos, que dificultan la valoración a largo plazo del resultado del tratamiento quirúrgico. La mejoría de la marcha y del control de esfínteres se produce antes que la mejoría de la función cognitiva. En el estudio de Raftopoulos y cols. ${ }^{61}$, la mejoría en el control de esfínteres se obtiene ya dentro de los primeros 9 días de la cirugía. En nuestros pacientes hemos objetivado que la mejoría en la marcha y en el control de esfínteres se produce de forma muy precoz, siendo evidente en la mayoría de los casos dentro de la primera semana después de la cirugía. A los dos meses, el 90\% de los pacientes ha mejorado la marcha, y a los 12 meses se obtiene el mayor grado de mejoría en las funciones mnésicas $^{61}$. Otros estudios han demostrado que la función cognitiva puede seguir mejorando, aunque de forma discreta, dentro del segundo año postquirúrgico ${ }^{23,40}$. Savolainen y cols. ${ }^{67}$ estudiaron el grado de mejoría postquirúrgica en pacientes con HCA idiopática a los 3, 12 meses y a los 5 años de la intervención. Estos autores observaron que a los 5 años se produce un decremento en el grado de mejoría en comparación con los resultados obtenidos a los 3 meses. Por tanto, parece ser que el intervalo de tiempo más apropiado para valorar los resultados de la cirugía sobre la función cognitiva sería entre los 6 y 12 meses de la intervención. Hasta el momento actual, en nuestro centro los controles postquirúrgicos se realizan entre los 6 y 9 meses de la colocación de la válvula.

\section{Propuesta de protocolo para la valoración clínica y neuropsicológica de la HCA}

\section{1) Tests breves de cribado}

En nuestro centro utilizamos el $\mathrm{MMSE}^{22}$ y el Frontal 
Assessment Battery ${ }^{17,65}$ como tests breves de cribaje en pacientes con sospecha de HCA. A pesar de que es bien conocido que el MMSE no define adecuadamente las alteraciones cognitivas de los pacientes con HCA, muchos grupos siguen incluyéndolo en su batería de estudio, ya que este test proporciona una medida rápida y global de la severidad de la alteración cognitiva. La puntuación máxima es de 30 puntos. Puntuaciones por debajo de 24 indican la presencia de una demencia. El Frontal Assessment Battery ${ }^{17,65}$ se ha diseñado para valorar la función del lóbulo frontal. Cada subtest se asocia a áreas específicas del lóbulo frontal, permitiendo valorar la severidad del síndrome disejecutivo. La puntuación máxima es de 18 puntos y se puede administrar en aproximadamente 10 minutos.

\section{2) Valoración clínica}

Los síntomas clínicos de la tríada de Hakim y Adams se valoran en nuestro centro mediante la escala de Hidrocefalia de Presión Normal (NPH), que fue publicada en 1991 por Sahuquillo y cols. ${ }^{66}$. Esta escala ordinal, permite determinar la severidad del cuadro clínico del paciente a través de puntuaciones que valoran de forma independiente el grado de afectación de la marcha, el control de esfínteres y la alteración cognitiva. La escala NPH tiene un rango de 3 - 15. La puntuación mínima de 3 corresponde a un paciente que no deambula y permanece siempre encamado o en sedestación, con incontinencia de ambos esfínteres y que se encuentra en una situación de mínima conciencia. La puntuación máxima de 15 , indica que el paciente presenta una marcha normal, no refiere alteraciones subjetivas a nivel cognitivo y muestra un control normal de esfínteres (Tabla 1).

Dado que las alteraciones motoras de los pacientes con una HCA van más allá de las alteraciones de la marcha, en nuestro centro cuantificamos la capacidad motora del enfermo mediante el denominado "Motor Performance Test” (MPT). El MPT es una escala semicuantitativa que valora seis actividades motoras potencialmente alteradas en estos pacientes : 1) tiempo requerido para levantarse de una silla, 2) subir y bajar repetidamente un peldaño de $23 \mathrm{~cm}$ de altura durante 10 segundos, 3) errores al realizar la marcha en tándem (pasos realizados fuera de una línea de dos metros, pasos realizados con los pies separados y número total de apoyos requeridos), 4) caminar 5 metros (con cuantificación del tiempo y número de pasos para determinar la velocidad media y la longitud media del paso), 5) equilibrio al apoyarse solo un pie y 6) número de pasos necesarios para realizar un giro de $180^{\circ}$. La puntuación mínima a conseguir con esta escala motora es "0" y la máxima 14, que corresponde a una actividad motora normal.
Tabla 1

Escala de Hidrocefalia de Presión Normal (NPH) para la valoración de la tríada clínica

Componentes de la escala

Puntuación

\section{Marcha}

Deambulación imposible $\quad 1$

Puede caminar con ayuda $\quad 2$

Inestabilidad. Caídas 3

Marcha anormal pero estable 4

Marcha normal

\section{Funciones cognitivas}

Estado vegetativo 1

Demencia grave 2

Problemas de memoria y cambio de carácter 3

Problemas de memoria 4

La familia o el paciente no manifiestan problemas 5 cognitivos

\section{Control de esfínteres}

Incontinencia urinaria y fecal 1

Incontinencia urinaria continua $\quad 2$

Incontinencia urinaria esporádica 3

Urgencia urinaria 4

Control normal de esfínteres 5

\section{3) Escalas funcionales}

Las tres escalas que se describen a continuación se utilizan para determinar el grado de dependencia del paciente para las actividades de la vida diaria:

1) El Rapid Disability Rating Scale-2 ${ }^{50}$ valora el grado de discapacidad del paciente. Esta escala incluye 18 ítems con cuatro posibles respuestas ( 0 - 3). La máxima puntuación es de 72, que corresponde a un paciente totalmente dependiente, y la mínima de 18 , que corresponde a un paciente que no necesita ayuda en las actividades de la vida diaria.

2) Una modificación de la Escala de Stein y Langfitt ${ }^{10}$ que incluye 6 grados de dependencia: una puntuación de 0 corresponde a un paciente capaz de trabajar, sin ningún déficit neurológico, y una puntuación de 5 hace referencia a un paciente que se encuentra en estado vegetativo (Tabla 2).

3) La Escala de actividades de la vida diaria ${ }^{20}$ valora el grado de ayuda que requiere el enfermo en 5 actividades de la vida diaria: movilidad, realizar compras, preparar la comida, realizar las tareas domésticas y manejar dinero. 
Tabla 2

Escala de Stein y Langfitt utilizada en nuestro centro para valorar el grado de dependencia del paciente en las AVD

Grado $0 \quad$ No déficit neurológico. Capaz de trabajar.

Grado 1 Déficit mínimo. Independiente en las actividades de la vida diaria.

Grado 2 Requiere alguna supervisión en casa.

Grado 3 Requiere supervisión permanente a pesar de una elevada capacidad funcional.

Grado 4 Dependiente en las actividades de la vida diaria.

Grado $5 \quad$ Vegetativo. Encamado.

AVD: Actividades de la vida diaria

\section{4) Evaluación neuropsicológica}

Dado que los pacientes con una HCA muestran una alteración de la memoria, de la atención, de la concentración, de la velocidad psicomotora y de las funciones ejecutivas, la evaluación neuropsicológica deberá tener en cuenta todos estos dominios ${ }^{18,19,76}$. En la Tabla 3 se resume el pro- tocolo utilizado en nuestro centro y se resaltan las pruebas más utilizadas en las series publicadas.

Para la evaluación de la memoria debe valorarse tanto la memoria visual como la memoria verbal. Para valorar la memoria verbal seleccionamos el Test de Aprendizaje Auditivo Verbal de Rey ${ }^{47}$, cuyo objetivo es el aprendizaje de una lista de palabras. Este test también valora la memoria diferida, cuando pedimos al paciente que repita al cabo de unos minutos la misma lista de palabras, y el reconocimiento del material aprendido. Para valorar la memoria visual inmediata y diferida utilizamos el subtest de Reproducción Visual I y II de la escala de memoria de Wechsler Revisada (WMS-R $)^{78}$. La memoria inmediata y la memoria de trabajo se pueden valorar mediante el subtest de dígitos directos e inversos de la WMS-R.

Para valorar la función del lóbulo frontal utilizamos el Test del Trazo ${ }^{64}$ y la fluencia verbal ${ }^{47}$. El Test del Trazo consta de dos partes. En la parte A, el paciente debe conectar, mediante líneas y de forma consecutiva, 25 números distribuidos al azar. La parte B es similar, pero en este caso el paciente debe unir números y letras de forma alternante (Figura 4). Estas pruebas valoran la atención visual sostenida, el rastreo visual, las habilidades grafomotoras, la secuenciación y la flexibilidad cognitiva. Existe una versión

Tabla 3

Protocolo de valoración clínica y neuropsicológica utilizado en los pacientes con una HCA

Tests de cribaje

Valoración clínica

Escalas funcionales

Valoración motora

Evaluación neuropsicológica

Memoria

Memoria visual
Memoria verbal inmediata
Aprendizaje verbal
Función frontal

Velocidad motora

Lenguaje

Praxias

Conducta
Mini Mental State Examination (MMSE)*

Frontal Assessment Battery (FAB)

Escala de Hidrocefalia de Presión Normal (NPH)

Rapid Disability Rating Scale-2 (RDRS-2)

Escala de Stein y Langfitt

Escala de Actividades de la vida diaria

Motor Performance Test

Wechsler Memory Scale-R*

Subtest de Dígitos del WAIS

Test de Aprendizaje Auditivo Verbal de Rey*

Test del Trazo A y B*

Fluencia verbal*

Purdue Pegboard Test

Test de Boston para el diagnóstico de la afasia

Test de Boston para el diagnóstico de la afasia

Frontal Behavioral Inventory

HCA: hidrocefalia crónica del adulto; * Tests neuropsicológicos más utilizados en las series publicadas 

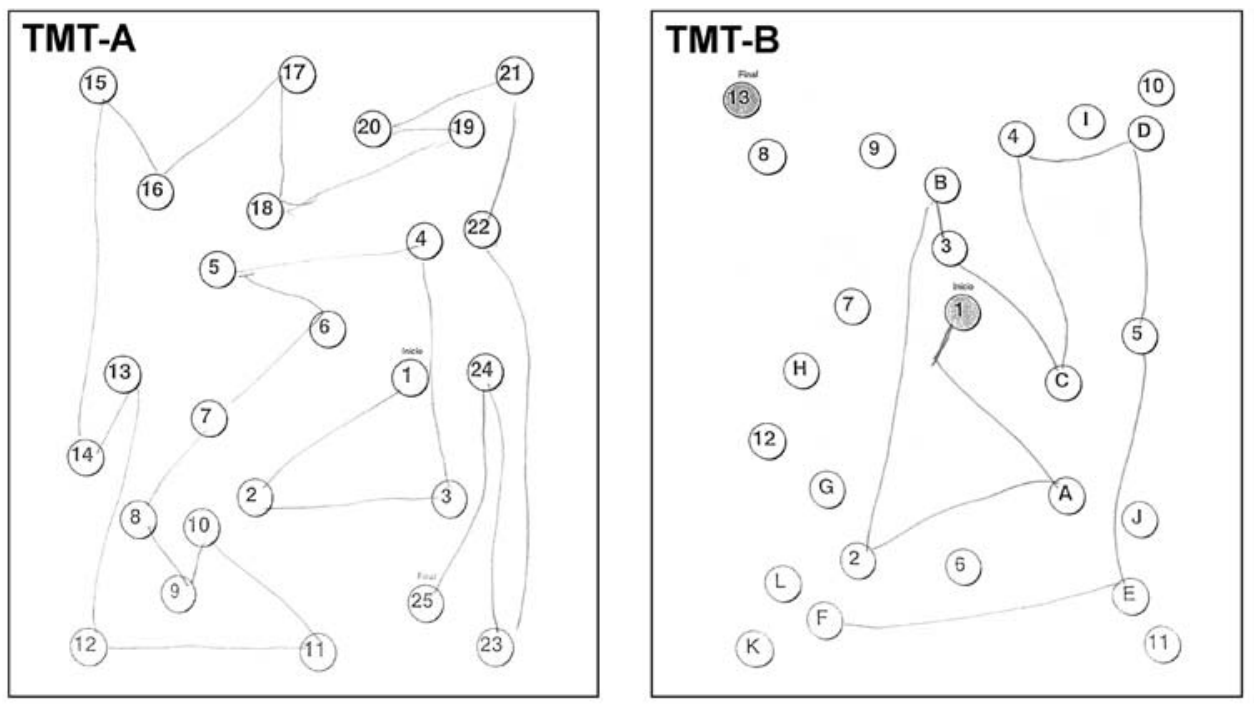

Figura 4. Test del trazo, parte $A$ y $B$, de un paciente con hidrocefalia crónica del adulto. En la parte A, el paciente ha unido, sin dificultades, los 25 números de forma consecutiva. En la parte $B$, se observa una incapacidad para seguir, de forma alternante, las secuencias de letras y números. adicional de esta prueba (Color Trails Test ${ }^{47}$ ), que puede utilizarse en pacientes sin escolarización. Esta versión sustituye las letras del abecedario por colores, quedando libre de la influencia del lenguaje y del nivel educativo del paciente. Al realizar una valoración neuropsicológica, es muy importante considerar y registrar el nivel educacional previo del enfermo, dado que en nuestro medio un número importante de los pacientes con una HCA idiopática presentan edades muy avanzadas y una baja escolarización. En la tarea de fluencia verbal fonética y semántica, se solicita al paciente que diga palabras que empiecen por la letra $\mathrm{S}$ y nombres de animales, respectivamente, en un intervalo de tiempo de un minuto ${ }^{47}$.

La velocidad y destreza motora manual se valoran mediante el Purdue Pegboard Test $t^{47}$. Esta prueba consiste en insertar el máximo número de clavijas en los orificios de un tablero durante 30 segundos. Primero se realiza con la mano

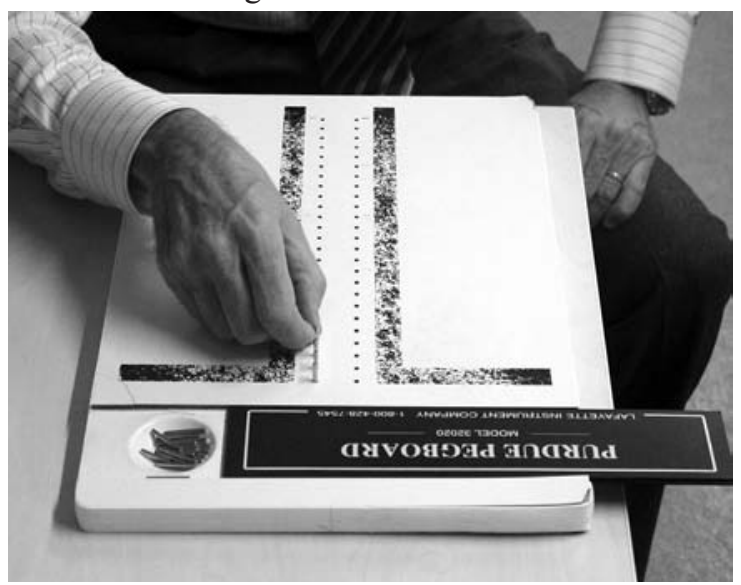

Figura 5. Purdue Pegboard Test, para la valoración de la velocidad y destreza motora manual. En esta prueba el paciente debe colocar el máximo número de clavijas en los orificios del tablero durante un máximo de 30 segundos. dominante y después con la no dominante (Figura 5).

Para poder descartar la presencia de un proceso neurodegenerativo de tipo cortical, como la enfermedad de Alzheimer, deberán incluirse pruebas para la valoración del síndrome afaso-apracto-agnósico, característico de este tipo de demencias. Para descartar la afasia, valoramos tanto la capacidad de denominación verbal como la comprensión de órdenes. El test de denominación de Boston ${ }^{27}$ valora la capacidad de denominación verbal por confrontación visual. Consta de 60 figuras de objetos, ordenados de menor a mayor dificultad, que el paciente debe denominar. Las dificultades inherentes a la utilización de un test tan extenso en ciertos pacientes ha llevado al desarrollo, en otros idiomas, de versiones reducidas de 15 y 30 ítems, que presentan una alta sensibilidad y especificidad para el diagnóstico de la Enfermedad de Alzheimer ${ }^{45,51,69}$. El análisis cualitativo del tipo de errores en la tarea de denominación nos permitirá distinguir entre problemas relacionados con una alteración del lenguaje, de los asociados a una alteración en el reconocimiento visual de los objetos (agnosia). La comprensión verbal se valora mediante la comprensión de órdenes del Test de Boston para el Diagnóstico de la Afasia ${ }^{27}$. Este mismo instrumento incluye un subtest que permite valorar la presencia de una apraxia ideomotora, mediante la realización de cinco movimientos transitivos (por ej. cómo usar un martillo) y cinco movimientos intransitivos (por ej. decir adiós) que el paciente debe realizar con los miembros superiores.

La administración de estas pruebas puede tener una duración aproximada de unos 60 - 90 minutos, dependiendo de la gravedad del deterioro cognitivo del paciente que se está evaluando.

\section{5) Valoración conductual}

En los pacientes con sospecha de HCA, además de la 
valoración neuropsicológica es conveniente realizar una valoración de potenciales alteraciones conductuales. El Frontal Behavioral Inventory ${ }^{41}$ es un cuestionario de 24 preguntas relacionadas con las manifestaciones conductuales más relevantes que se observan en la demencia frontal, como la apatía, la falta de espontaneidad, la irritabilidad, la fatigabilidad, etc. Cada pregunta se puntúa según la intensidad del cambio en una escala de 0 a 3 puntos.

\section{Consideraciones Finales}

Las diferentes series publicadas sobre los resultados del tratamiento quirúrgico en los pacientes con una HCA muestran resultados muy dispares. Esta variabilidad se debe a la inclusión de pacientes con HCA de diversas etiologías, a la utilización de protocolos diagnósticos y terapéuticos muy distintos y a la coexistencia en muchos casos de otras causas de demencia. El estudio neuropsicológico de estos pacientes puede contribuir, de forma importante, al diagnóstico de la HCA. Los pacientes con HCA presentan un patrón de afectación de tipo fronto-subcortical, en ausencia de síntomas corticales, como afasia, apraxia o agnosia. La detección de este patrón y la ausencia de los síntomas corticales mencionados nos va a permitir diferenciar esta patología de otras demencias neurodegenerativas.

No existe en la actualidad un sistema estandarizado para la valoración de la situación basal de estos pacientes y la cuantificación de sus cambios después del tratamiento quirúrgico. Sin embargo, es importante incluir escalas funcionales para determinar el grado de incapacidad o dependencia del paciente en las actividades de la vida diaria, así como una valoración de su funcionamiento cognitivo y conductual. Tampoco existe un consenso respecto al momento óptimo para realizar dicha valoración. El momento idóneo para la valoración postquirúrgica sería entre los 6 y 12 meses después de la intervención. Después de este intervalo de tiempo se añadirían otros factores de comorbilidad que confunden los resultados y que no guardan relación con la cirugía.

\section{Agradecimientos}

Este estudio ha sido financiado parcialmente por el Fondo de Investigación Sanitaria de la Seguridad Social (FIS, beca 07/0681), por el Institiut Fundació de Recerca Vall d'Hebrón (beca predoctoral de B. Benejam), y respaldado por la Generalitat de Catalunya (2005SGR00411). Agradecemos la constante colaboración e inestimable ayuda de las enfermeras del Servicio de Neurocirugía del Hospital Universitario Valle Hebrón, especialmente a Maria Soledad Armengol, Maria Jesús Peñarrubia, Eva Corral y Maria Asunción Muns.

\section{Bibliografía}

1. Abbruzzese, M., Scarone, S., Colombo, C.: Obsessivecompulsive symptomatology in normal pressure hydrocephalus: a case report. J.Psychiatry Neurosci. 1994; 19: 378-380.

2. Adams, R.D., Fisher, C.M., Hakim, S., et al: symptomatic occult hydrocephalus with "normal" cerebrospinal-fluid pressure. A treatable syndrome. N.Engl.J.Med. 1965; 273: 117-126.

3. American Psychiatric Association: DSM-IV: Manual Diagnóstico y Estadístico de los Trastornos Mentales, ed $1^{\mathrm{a}}$ ed. Barcelona: Masson, S.A., 1995.

4. Bech, R.A., Waldemar, G., Gjerris, F., et al.: Shunting effects in patients with idiopathic normal pressure hydrocephalus; correlation with cerebral and leptomeningeal biopsy findings. Acta Neurochir. (Wien.) 1999; 141: 633-639.

5. Bech-Azeddine, R., Waldemar, G., Knudsen, G.M., et al.: Idiopathic normal-pressure hydrocephalus: evaluation and findings in a multidisciplinary memory clinic. Eur.J.Neurol. 2001; 8: 601-611.

6. Benzel, E.C., Pelletier, A.L., Levy, P.G.: Communicating hydrocephalus in adults: prediction of outcome after ventricular shunting procedures. Neurosurgery 1990; 26: 655660 .

7. Black, P.M.: Idiopathic normal-pressure hydrocephalus. Results of shunting in 62 patients. J.Neurosurg. 1980; 52: 371377.

8. Bloom, K.K., Kraft, W.A.: Paranoia--an unusual presentation of hydrocephalus. Am. J. Phys. Med. Rehabil. 1998; 77: 157-159.

9. Boon, A.J., Tans, J.T., Delwel, E.J., et al.: Dutch normalpressure hydrocephalus study: prediction of outcome after shunting by resistance to outflow of cerebrospinal fluid. J. Neurosurg. 1997; 87: 687-693.

10. Borgesen, S.E.: Conductance to outflow of CSF in normal pressure hydrocephalus. Acta Neurochir. (Wien.) 1984; 71: 1-45.

11. Bret, P., Guyotat, J., Chazal, J.: Is normal pressure hydrocephalus a valid concept in 2002? A reappraisal in five questions and proposal for a new designation of the syndrome as "chronic hydrocephalus". J. Neurol. Neurosurg. Psychiatry 2002; 73: 9-12.

12. Caltagirone, C., Gainotti, G., Masullo, C., et al.: Neurophysiological study of normal pressure hydrocephalus. Acta Psychiatr. Scand. 1982; 65: 93-100.

13. Caruso, R., Cervoni, L., Vitale, A.M., et al.: Idiopathic normal-pressure hydrocephalus in adults: result of shunting correlated with clinical findings in 18 patients and review of the literature. Neurosurg. Rev. 1997; 20: 104-107.

14. Chakravarty, A.: Unifying concept for Alzheimer's disease, vascular dementia and normal pressure hydrocephalus - a hypothesis. Med. Hypotheses 2004; 63: 827-833.

15. Crowell, R.M., Tew, J.M., Jr., Mark, V.H.: Aggressive 
dementia associated with normal pressure hydrocephalus. Report of two unusual cases. Neurology 1973; 23: 461-464.

16. Devito, E.E., Pickard, J.D., Salmond, C.H., et al.: The neuropsychology of normal pressure hydrocephalus (NPH). Br. J. Neurosurg. 2005; 19: 217-224.

17. Dubois, B., Slachevsky, A., Litvan, I., et al: The FAB: a Frontal Assessment Battery at bedside. Neurology 2000; 55 : 1621-1626.

18. Duinkerke, A., Williams, M.A., Rigamonti, D., et al.: Cognitive recovery in idiopathic normal pressure hydrocephalus after shunt. Cogn Behav. Neurol. 2004; 17: 179-184.

19. Farace, E., Shaffrey, M.E.: Value of neuropsychological information for improved understanding of the patient with normal-pressure hydrocephalus. J. Neurosurg. 2005; 102: 971972.

20. Fillenbaum, G.G.: Screening the elderly. A brief instrumental activities of daily living measure. J. Am. Geriatr. Soc. 1985; 33: 698-706.

21. Fisher, C.M.: Hydrocephalus as a cause of disturbances of gait in the elderly. Neurology 1982; 32: 1358-1363.

22. Folstein, M.F., Folstein, S.E., McHugh, P.R.: "Minimental state". A practical method for grading the cognitive state of patients for the clinician. J. Psychiatr. Res. 1975; 12: 189-198.

23. Friedland, R.P.: 'Normal'-pressure hydrocephalus and the saga of the treatable dementias. JAMA 1989; 262: 25772581 .

24. George, A.E., Holodny, A., Golomb, J., et al.: The differential diagnosis of Alzheimer's disease. Cerebral atrophy versus normal pressure hydrocephalus. Neuroimaging Clin. N. Am. 1995; 5: 19-31.

25. Golomb, J., de Leon, M.J., George, A.E., et al.: Hippocampal atrophy correlates with severe cognitive impairment in elderly patients with suspected normal pressure hydrocephalus. J. Neurol. Neurosurg. Psychiatry 1994; 57: 590-593.

26. Golomb, J., Wisoff, J., Miller, D.C., et al.: Alzheimer's disease comorbidity in normal pressure hydrocephalus: prevalence and shunt response. J. Neurol. Neurosurg. Psychiatry 2000; 68: 778-781.

27. Goodglass, H., Kaplan, E.: Evaluación de la afasia y de trastornos relacionados. Madrid: Editorial Médica Panamericana, 1996.

28. Graff-Radford, N.R., Godersky, J.C.: Normal-pressure hydrocephalus. Onset of gait abnormality before dementia predicts good surgical outcome. Arch. Neurol. 1986; 43: 940942.

29. Graff-Radford, N.R., Godersky, J.C., Jones, M.P.: Variables predicting surgical outcome in symptomatic hydrocephalus in the elderly. Neurology 1989; 39: 1601-1604.

30. Gustafson, L., Hagberg, B.: Recovery in hydrocephalic dementia after shunt operation. J. Neurol. Neurosurg. Psychiatry 1978; 41: 940-947.

31. Hakim, S.: Algunas observaciones sobre la presión del L.C.R. Síndrome hidrocefálico en el adulto con "presión normal" del L.C.R. 1964. Facultad de Medicina, Universidad Javierana. Bogota.

Ref Type: Thesis/Dissertation

32. Hakim, S., Adams, R.D.: The special clinical problem of symptomatic hydrocephalus with normal cerebrospinal fluid pressure. Observations on cerebrospinal fluid hydrodynamics. J. Neurol. Sci. 1965; 2: 307-327.

33. Hebb, A.O., Cusimano, M.D.: Idiopathic normal pressure hydrocephalus: a systematic review of diagnosis and outcome. Neurosurgery 2001; 49: 1166-1184.

34. Helkala, E.L., Laulumaa, V., Soininen, H., et al.: Recall and recognition memory in patients with Alzheimer's and Parkinson's diseases. Ann. Neurol. 1988; 24: 214-217.

35. Holodny, A.I., George, A.E., de Leon, M.J., et al: Focal dilation and paradoxical collapse of cortical fissures and sulci in patients with normal-pressure hydrocephalus. J. Neurosurg. 1998; 89: 742-747.

36. Holodny, A.I., Waxman, R., George, A.E., et al.: MR differential diagnosis of normal-pressure hydrocephalus and Alzheimer disease: significance of perihippocampal fissures. AJNR Am. J. Neuroradiol. 1998; 19: 813-819.

37. Huber, S.J., Shuttleworth, E.C., Paulson, G.W., et al.: Cortical vs subcortical dementia. Neuropsychological differences. Arch. Neurol. 1986; 43: 392-394.

38. Iddon, J.L., Pickard, J.D., Cross, J.J., et al.: Specific patterns of cognitive impairment in patients with idiopathic normal pressure hydrocephalus and Alzheimer's disease: a pilot study. J. Neurol. Neurosurg. Psychiatry 1999; 67: 723732 .

39. Jack, C.R., Jr., Petersen, R.C., O'Brien, P.C., et al.: MRbased hippocampal volumetry in the diagnosis of Alzheimer's disease. Neurology 1992: 42: 183-188.

40. Kaye, J.A., Grady, C.L., Haxby, J.V., et al.: Plasticity in the aging brain. Reversibility of anatomic, metabolic, and cognitive deficits in normal-pressure hydrocephalus following shunt surgery. Arch. Neurol. 1990; 47: 1336-1341.

41. Kertesz, A., Davidson, W., Fox, H.: Frontal behavioral inventory: diagnostic criteria for frontal lobe dementia. Can. J. Neurol. Sci. 1997; 24: 29-36.

42. Kesslak, J.P., Nalcioglu, O., Cotman, C.W.: Quantification of magnetic resonance scans for hippocampal and parahippocampal atrophy in Alzheimer's disease. Neurology 1991; 41: 51-54.

43. Kitagaki, H., Mori, E., Ishii, K., et al.: CSF spaces in idiopathic normal pressure hydrocephalus: morphology and volumetry. AJNR Am. J. Neuroradiol. 1998; 19: 1277-1284.

44. Klinge, P., Marmarou, A., Bergsneider, M., et al.: Outcome of shunting in idiopathic normal-pressure hydrocephalus and the value of outcome assessment in shunted patients. Neurosurgery 2005; 57: S40-S52.

45. Lansing, A.E., Ivnik, R.J., Cullum, C.M., et al.: An empirically derived short form of the Boston naming test. 
Arch. Clin. Neuropsychol. 1999; 14: 481-487.

46. Larsson, A., Wikkelso, C., Bilting, M., et al.: Clinical parameters in 74 consecutive patients shunt operated for normal pressure hydrocephalus. Acta Neurol. Scand. 1991; 84: 475-482.

47. Lezak, M.: Neuropsychological assessment, ed $3^{\circ}$ edition. New York: Oxford University Press, 1995.

48. Lindqvist, G., Andersson, H., Bilting, M., et al.: Normal pressure hydrocephalus: psychiatric findings before and after shunt operation classified in a new diagnostic system for organic psychiatry. Acta Psychiatr. Scand. Suppl 1993; 373: 18-32.

49. Lindqvist, G., Malmgren, H.: Organic mental disorders as hypothetical pathogenetic processes. Acta Psychiatr. Scand. Suppl 1993; 373: 5-17.

50. Linn, M.W., Linn, B.S.: The rapid disability rating scale-2. J. Am. Geriatr. Soc. 1982; 30: 378-382.

51. Mack, W.J., Freed, D.M., Williams, B.W., et al.: Boston Naming Test: shortened versions for use in Alzheimer's disease. J. Gerontol. 1992; 47: 154-158.

52. Marmarou, A., Young, H.F., Aygok, G.A., et al.: Diagnosis and management of idiopathic normal-pressure hydrocephalus: a prospective study in 151 patients. J. Neurosurg. 2005; 102: 987-997.

53. Mattis, S.: Mental status examination for organic mental syndrome in the elderly patient, in Karasu T (ed): Geriatric Psychiatry. New York: Grune \& Stratton, 1976; pp 77-121

54. Miyoshi, N., Kazui, H., Ogino, A., et al.: Association between cognitive impairment and gait disturbance in patients with idiopathic normal pressure hydrocephalus. Dement. Geriatr. Cogn Disord.2005; 20: 71-76.

55. Nagaratnam, N., Verma, S., Nagaratnam, K., et al.: Psychiatric and behavioural manifestations of normal-pressure hydrocephalus. Br. J. Clin. Pract. 1994; 48: 122-124.

56. Pinner, G., Johnson, H., Bouman, W.P., et al.: Psychiatric manifestations of normal-pressure hydrocephalus: a short review and unusual case. Int. Psychogeriatr. 1997; 9: 465-470.

57. Poca, M.A., Mataro, M., Del Mar, M.M., et al.: Is the placement of shunts in patients with idiopathic normal-pressure hydrocephalus worth the risk? Results of a study based on continuous monitoring of intracranial pressure. J. Neurosurg. 2004; 100: 855-866.

58. Poca, M.A., Mataro, M., Matarin, M., et al.: Good outcome in patients with normal-pressure hydrocephalus and factors indicating poor prognosis. J. Neurosurg. 2005; 103 : 455-463.

59. Poca, M.A., Sahuquillo, J., Mataro, M.: [Update on diagnosis and treatment of normotensive hydrocephalus (chronic hydrocephalus of the adult)]. Neurologia 2001; 16: 353-369.

60. Power, C., Selnes, O.A., Grim, J.A., et al.: HIV Dementia Scale: a rapid screening test. J. Acquir. Immune.
Defic. Syndr. Hum. Retrovirol. 1995; 8: 273-278.

61. Raftopoulos, C., Deleval, J., Chaskis, C., et al.: Cognitive recovery in idiopathic normal pressure hydrocephalus: a prospective study. Neurosurgery 1994; 35: 397-404.

62. Raftopoulos, C., Massager, N., Baleriaux, D., et al.: Prospective analysis by computed tomography and long-term outcome of 23 adult patients with chronic idiopathic hydrocephalus. Neurosurgery 1996; 38: 51-59.

63. Reisberg, B., Ferris, S.H., de Leon, M.J., et al.: The Global Deterioration Scale for assessment of primary degenerative dementia. Am. J. Psychiatry 1982; 139: 1136-1139.

64. Reitan, R.M.: The relation of the trail making test to organic brain damage. J. Consult Psychol 1955; 19: 393-394.

65. Rodriguez, d.A., Catalan Alonso, M.J., Carrasco, M.L.: [FAB: a preliminar Spanish application of the frontal assessment battery to 11 groups of patients]. Rev. Neurol. 2003; 36: 605-608.

66. Sahuquillo, J., Rubio, E., Codina, A., et al.: Reappraisal of the intracranial pressure and cerebrospinal fluid dynamics in patients with the so-called "normal pressure hydrocephalus" syndrome. Acta Neurochir. (Wien.) 1991; 112: 50-61.

67. Savolainen, S., Hurskainen, H., Paljarvi, L., et al.: Five-year outcome of normal pressure hydrocephalus with or without a shunt: predictive value of the clinical signs, neuropsychological evaluation and infusion test. Acta Neurochir. (Wien.) 2002; 144: 515-523.

68. Savolainen, S., Paljarvi, L., Vapalahti, M.: Prevalence of Alzheimer's disease in patients investigated for presumed normal pressure hydrocephalus: a clinical and neuropathological study. Acta Neurochir. (Wien.) 1999; 141: 849-853.

69. Serrano, C., Allegri, R.F., Drake, M., et al.: [A shortened form of the Spanish Boston naming test: a useful tool for the diagnosis of Alzheimer's disease]. Rev. Neurol. 2001; 33: 624-627.

70. Stambrook, M., Cardoso, E., Hawryluk, G.A., et al.: Neuropsychological changes following the neurosurgical treatment of normal pressure hydrocephalus. Arch. Clin. Neuropsychol. 1988; 3: 323-330.

71. Thomas, G., McGirt, M.J., Woodworth, G., et al.: Baseline neuropsychological profile and cognitive response to cerebrospinal fluid shunting for idiopathic normal pressure hydrocephalus. Dement. Geriatr. Cogn Disord. 2005; 20: 163168.

72. Thomsen, A.M., Borgesen, S.E., Bruhn, P., et al.: Prognosis of dementia in normal-pressure hydrocephalus after a shunt operation. Ann. Neurol. 1986; 20: 304-310.

73. Tullberg, M., Hellstrom, P., Piechnik, S.K., et al.: Impaired wakefulness is associated with reduced anterior cingulate CBF in patients with normal pressure hydrocephalus. Acta Neurol. Scand. 2004; 110: 322-330.

74. van Harten, B., Courant, M.N., Scheltens, P., et al.: Validation of the HIV Dementia Scale in an elderly cohort of patients with subcortical cognitive impairment caused by 
subcortical ischaemic vascular disease or a normal pressure hydrocephalus. Dement. Geriatr. Cogn Disord. 2004; 18: 109114.

75. Vanneste, J., Augustijn, P., Dirven, C., et al.: Shunting normal-pressure hydrocephalus: do the benefits outweigh the risks? A multicenter study and literature review. Neurology 1992; 42: 54-59.

76. Vanneste, J.A.: Diagnosis and management of normalpressure hydrocephalus. J. Neurol. 2000; 247: 5-14.

77. Vassilouthis, J.: Poor prognosis/good outcome in patients with NPH. J. Neurosurg. 12006; 04: 986-988.

78. Wechsler, D.: A standardized memory scale for clinical use. J. Psychol 1945; 19: 87-95.

\section{Glosario de Términos Neuropsicológicos}

- Fabulación: falsificación del recuerdo. El paciente evoca una información o un recuerdo que sólo existe en su fantasía.

- Alteraciones Visuoconstructivas: dificultad para planificar y ejecutar actos motores implicados en la realización de tareas como ensamblar, construir o dibujar, en ausencia de alteraciones motoras.

- Alteraciones Visuoespaciales: trastornos que se carac- terizan por una dificultad en la percepción o identificación espacial de los objetos o estímulos presentados, como por ejemplo, encontrar un producto en el supermercado o colocar las manecillas del reloj en la hora señalada.

- Funciones Ejecutivas: conjunto de habilidades cognitivas que permiten la anticipación y el establecimiento de metas, la formación de planes y programas, el inicio de las actividades y operaciones mentales, la autorregulación de las tareas y la habilidad de llevarlas a cabo eficientemente.

- Memoria de Trabajo: sistema cerebral que proporciona almacenamiento temporal para ejecutar funciones cognitivas complejas, como por ejemplo, realizar cálculos mentales o decir los meses del año al revés.

Benejam, B.; Poca, M.A.; Junqué, C.; Solana, E.; Sahuqui1lo, J.: Alteraciones cognitivas en pacientes con hidrocefalia crónica del adulto ("normotensiva"). Propuesta de un protocolo para su evaluación clínica . Neurocirugía 2008; 19: 309-321.

Correspondencia postal: $\mathrm{M}^{\mathrm{a}}$ Antonia Poca. Servicio de Neurocirugía. Hospital Universitario Valle Hebrón. Paseo Valle Hebrón 119-129. 08035 Barcelona. 